\title{
Repeating phrases across unrelated narratives: Evidence of text repetition effects
}

\author{
Celia M. Klin and Angela S. Ralano \\ State University of New York, Binghamton, New York \\ AND \\ KRISTIN M. WeingaRTNER \\ Hofstra University, Hempstead, New York
}

\begin{abstract}
Research has shown that text repetition effects are limited to conditions in which the context remains consistent across the two processing episodes, particularly when readers are focused on comprehension. Despite this, we found evidence of transfer effects across unrelated narratives. In a repeated condition, an ambiguous phrase appeared in two consecutive stories. In Story A, the phrase was presented in a sarcasm-biasing context, and in Story B, the phrase was presented in a neutral context. The pattern of findings from an offline measure (Experiment 1 ) and a reading time measure (Experiments 2, 3, and 4) indicated that participants were more likely to interpret the phrase in Story B as sarcastic in the repeated version than in a nonrepeated version, in which the phrase was absent from Story A. We conclude that during the reading of Story B, the phrase was reactivated from memory, even though the two stories were unrelated.
\end{abstract}

An important goal for a theory of discourse comprehension is to determine what information is included in a reader's memory representation. One of the ways this question has been addressed has been through studies of rereading, in which participants are asked to read a text two times. The logic of rereading studies is that only information that is included in a reader's memory representation from the original reading can facilitate rereading. Facilitation, defined as an increase in speed during a second reading or improved performance on a secondary task such as a probe task, is commonly referred to as a rereading benefit or text repetition effect (see, e.g., Carr, Brown, \& Charalambous, 1989; Levy \& Burns, 1990; Raney \& Rayner, 1995; Raney, Therriault, \& Minkoff, 2000).

An interesting finding from a large number of studies is that repetition effects are often limited to conditions in which the context remains consistent across the two processing episodes. Consider a few examples: Levy et al. (1995) found rereading benefits, measured by shorter reading times on the second reading, in a related condition in which two passages came from the same novel and, thus, shared characters, a story line, and a significant number of content words. In contrast, they found no rereading benefit in a condition in which the two passages shared neither characters nor a theme, but shared the same number of content words as the related condition. Similarly, Levy and Burns (1990) presented multiparagraph passages that were identical across two readings, had the individual paragraphs reordered, had the sentences reordered, or had the words reordered. Although there were rereading benefits for the identical condition and the paragraph-reordered condition, there were limited rereading benefits for the sentence-reordered condition and no rereading benefits for the word-reordered condition. And finally, using ERPs as the dependent measure, Besson and Kutas (1993) found repetition effects (indicated by a decrease in N400 amplitude during a second reading) when words were presented a second time in their original sentence frame but not when they were presented in a new sentence.

Studies using word lists have also found that repetition benefits tend to be limited to conditions in which the context remains consistent across the two processing episodes. For example, using a perceptual identification task, Levy and Kirsner (1989) found a benefit for words that were both studied and tested as a part of a word list, but found no benefit for words that were originally seen in the context of a text and subsequently identified in a word list. Oliphant (1983) used a lexical decision task and found a reprocessing benefit when words were repeated within the lexical decision task, but found no reprocessing benefit when the words were first processed as part of the instructions for the experiment. And Jacoby (1983) presented a test list that had either $90 \%$ of the words in common with the study list or $10 \%$ of the words in common, and found significantly reduced repetition effects when there was only $10 \%$ overlap. Similarly, MacLeod (1989) found reduced priming effects in a word-stem completion task when the words had originally been encountered in a connected discourse rather than studied as a part of a word list.

C.M. Klin, cklin@binghamton.edu 
Why might rereading transfer benefits be limited in this way? According to an episodic account (e.g., Tenpenny, 1995), repetition priming is the result of the reactivation of the initial processing episode (for similar accounts, see also Hayman \& Jacoby, 1989, and Jacoby, Baker, \& Brooks, 1989). Consistent with this, Levy et al. (1995) concluded that the memory representation of a word is contextually bound, or holistic (Levy \& Kirsner, 1989), and therefore only when that context is reactivated will there be any rereading benefits. Similarly, Masson and Freedman (1990) argued that repetition effects are dependent on repeating a word's original encoding context. Raney (2003) made similar arguments, but with reference to the idea of a situation model: "The situation model binds the surface features and the textbase to the text representation. The more complete the situation model, the more tightly glued the surface features and textbase become to the text representation" (p. 20).

Although most rereading studies provide evidence that is consistent with some sort of an episodic, or contextdependent, account, there are some exceptions. For example, although MacLeod (1989) found greater priming effects in a word-stem completion task when the words had originally been studied on a word list, there was a significant priming effect even when the words had originally been encountered as part of a text. Similarly, Carr et al. (1989) found evidence of abstract, or contextindependent, repetition effects when participants read paragraphs aloud. The words of the paragraph were presented in either a normal format or a scrambled format, and during rereading, the paragraphs were presented in their original format or in the alternative format. Carr et al. found repetition effects, measured by faster reading times during the second reading, in both conditions. That is, a repetition of the original word order was not necessary, suggesting that the representation of the words was not bound to the text representation. In contrast with the episodic account, Carr et al. concluded that repetition effects can be based on the priming of some type of abstract word representation, such as a logogen (Morton, 1969); the logogen is accessed and activated during the first presentation, lowering its input threshold and making access to it faster during the second presentation, regardless of the context.

How might these findings be explained in light of the extensive support for the episodic account? Carr and Brown (1990) offered a level-of-focal-attention hypothesis: When readers focus on comprehension, as they usually do, rather than on the individual words that make up a sentence or a passage, there should be a large contribution from text-level (i.e., episodic), or context-dependent, processing (e.g., Levy et al., 1995). However, if readers focus their attention on processing individual lexical items, there will be a large contribution from lexical, or context-independent, processing. Consistent with this, in the Carr et al. (1989) experiment, where repetition effects were found even when the format (i.e., normal vs. scrambled) was changed from the first to the second reading, not only had participants been instructed to read out loud, but they were instructed to read quickly and to clearly enunciate the words. This placed special emphasis on lexical processing, and in turn, led to context-independent processing.

Raney (2003) made similar arguments in his context-dependent representation model. He argued that the situation model is represented in a context-dependent manner, whereas surface information and textbase information (van Dijk \& Kintsch, 1983) is represented abstractly, in a context-independent manner. Because of this, Raney predicted that repetition effects should be context independent if a situation model has not been constructed - for example, when readers ignore the meaning of a text and focus on the individual lexical itemsand context dependent when there is a well-developed situation model.

One source of support for the ideas posited in the levelof-focal-attention hypothesis and in the context-dependent representation model is a finding from Carlson, Alenjano, and Carr (1991), who explicitly examined the influence of readers' focus of attention on repetition benefits. They replicated the Carr et al. (1989) experiment but used two different sets of instructions: One group of participants was told to ignore the meaning of the paragraphs and to concentrate on the individual words, and a second group was told to focus on the meaning of the paragraphs. When readers ignored the text meaning, Carlson et al. found repetition effects even when the format changed from the first presentation to the second, replicating the results of Carr et al. However, when readers focused on the text meaning, there were no repetition effects when the format changed across the two readings (see also Levy \& Burns, 1990). Thus, as predicted, the nature of readers' memory representation appears to be dependent, at least in part, on the way in which readers allocate their attentional resources.

Although this study demonstrates that there is an episodic component to the readers' text representation, at least when they are reading for comprehension, in the present set of experiments, we ask whether there are any conditions in which rereading benefits are found across unrelated stories, despite the change in context across the two processing episodes. We ask this even though the existing literature suggests that the answer is no (e.g., Levy et al., 1995; Raney, 2003): The context-dependent representation model predicts that when a situation model has been formed, repetition effects should be limited to "conceptually similar linguistic contexts" (p. 26). And Levy et al. found no evidence of repetition effects when two texts were thematically unrelated, even though they had many content words in common. However, there are both theoretical and empirical reasons to suspect that there should be a set of conditions under which a text input from one story serves to reactivate concepts from an unrelated story. First, given a memory-based text processing view (e.g., Gerrig \& McKoon, 1998; McKoon \& Ratcliff, 1995), such as Myers and O'Brien's (1998) resonance model, each text input should be able to reactivate related information from all of long-term memory - not only the episodic text representation, but also related information in general world knowledge (e.g., O'Brien \& Albrecht, 1992; Rizzella \& O'Brien, 2002). That is, related concepts 
from all of memory should resonate in parallel (e.g., Gillund \& Shiffrin, 1984; Hintzman, 1986; Ratcliff, 1978) as a function of the number of semantic and conceptual features that overlap with the current segment of text. Therefore, if a reader's knowledge base includes concepts from previous narratives, and if the current segment of text overlaps sufficiently with those concepts, a rereading effect should be found.

In addition to this theoretical justification for the prediction of a rereading effect across unrelated passages, it is interesting to consider a finding from Levy et al. (1995). In their word overlap condition, in which two texts shared a substantial number of words but were thematically unrelated, there was actually evidence of a small slowdown during reading of the second text, in comparison with an unrelated control. Although the reason for this negative transfer effect is not entirely clear, the finding raises the possibility that information from the original reading was retrieved from memory during the second reading.

To investigate whether there are any conditions in which rereading effects occur across unrelated passages, we attempted to create a set of passages in which repetition effects should be most likely to be found. In order to do this, we examined comprehension of a salient ambiguous phrase that was repeated across two passages. Consider the sample item in the Appendix. First, note that although Story A and Story B made up a single experimental item, participants believed that the stories were independent. This belief was reinforced by a quasirandom ordering of experimental and of filler passages, which meant that there was no obvious pattern for participants to discern. In addition, Story A and Story B were semantically unrelatedthey did not share a story line, characters, or a setting.

Our question involved the processing of the critical phrase in Story B. The phrase is ambiguous; in the sample passage, it is unclear whether Brian is being sincere or sarcastic. However, in the repeated version of Story A, the context clearly biases a sarcastic interpretation of the phrase. The logic of the design used in the experiments reported here is as follows: Assuming there is a rereading effect, participants will reactivate the critical phrase from Story A, as well as their interpretation of it, when they read the critical phrase in Story B in the repeated version. Thus, participants should be more likely to interpret the critical phrase in Story B as sarcastic in the repeated version than in the nonrepeated version. And consequently, in Experiment 1, there should be more "true" responses to the true-false statement in the repeated version because the statement (e.g., "Brian did not appreciate Laura's advice") is consistent with a sarcastic interpretation of the critical phrase.

To create a set of stimuli for which repetition effects were most likely to be found across unrelated texts, we examined the repetition of phrases, rather than individual words. Phrases should be more salient, and thus more memorable, than individual words, simply because they are longer. In addition, we selected phrases that should be particularly salient - they are in quotation marks and they are sarcastic. However, despite the probable salience of the repeated information, the design was relatively con- servative. We assume that without any biasing context, a sincere interpretation of the critical phrase in Story B should be the default, or dominant, interpretation. Therefore, to find a difference between versions, in the repeated version, not only must the representation of the phrase be reactivated from memory during the reading of Story B, it must have a sufficient impact on comprehension to bias a subordinate, sarcastic, interpretation, despite the lack of any supporting sarcastic context in Story B itself.

\section{EXPERIMENT 1}

In Experiment 1, we examined repetition effects across two unrelated stories. To maximize the probability of finding a rereading effect, we examined the influence of repeated phrases, rather than repeated words. The other way this design differs from previous investigations is the dependent measure. The majority of past studies of repetition effects have examined the time to process a lexical item during a second presentation-during a lexical decision task, a naming task, or simply during rereading; the logic is that any reactivation of the word from the initial processing episode should speed responding (e.g., Raney \& Rayner, 1995). However, in the present experiment, comparing reading times on the critical phrase across the two presentations would not have been appropriate. First, the phrase was unambiguous in Story A and ambiguous in Story B, a change that in and of itself should lead to differences in reading times. Second, in contrast to encountering a repeated word, encountering a repeated phrase might be surprising to readers, resulting in a slowdown in processing during Story B. Third, assuming that repetition effects across unrelated texts are likely to be modest in size, it was critical to find a dependent measure that would be as sensitive as possible. Given these considerations, instead of examining the influence of the first reading on the speed of the second reading, we examined the influence of the first reading on the comprehension of the second reading.

More specifically, in Experiment 1, a true-false statement was presented at the end of Story B that was consistent with a sarcastic interpretation of the critical phrase. In contrast with Experiments 2, 3, and 4, in which we used an online reading time measure, in Experiment 1 we began with a question-answering task in order to make sure that the materials were sensibly constructed. We wanted to determine whether, at least when asked an explicit question, readers would be more likely to judge the critical phrase in Story B as sarcastic when it had been used sarcastically in Story A than when it had not.

\section{Method}

Participants. A total of 58 undergraduates at the State University of New York at Binghamton participated as part of the requirement for an introductory psychology course. The data were eliminated from 4 participants who did not follow instructions. Therefore, our analyses were based on the data from 54 participants.

Materials. There were 14 experimental items, with each item consisting of a pair of unrelated stories, Story A and Story B (see the Appendix). There were two versions of Story A: repeated and nonrepeated. Both versions began with an introduction that de- 
scribed an interaction between two characters. This was followed by a critical information section. In the repeated version of this section, one character, the message writer, felt negatively about the interaction. Because of this, the message writer left a sarcastic note for the other character, the addressee. The sarcastic note consisted of two sentences (e.g., "Thanks so very much for all your advice. Sure is nice to be treated like a 10 year old."). The first sentence was the critical phrase that was repeated in Story B. This sentence was written so that if it appeared in isolation, without context, it would be ambiguous. However, to ensure that readers interpreted this phrase sarcastically in Story A, we made the sentence following the critical phrase clearly sarcastic. In addition, the sentence that preceded the critical phrase explicitly stated that the message writer was angry or unhappy (e.g., "She wrote an angry note to Eric telling him she always remembered to take out the garbage").

In the nonrepeated version of Story A, no information was given in the critical information section regarding how the interaction from the introduction was perceived by the message writer. Furthermore, the critical phrase was not included. In its place, the message writer left a note that was unambiguous and sincere (e.g., "I've left a pot of soup cooking on the stove on low heat. Can you turn it off at 10:00?"). The critical information sections from Story A were matched for length across the two versions. Finally, the conclusion provided a two-sentence story ending. The conclusion was identical in both versions and was neutral with regard to the critical phrase.

Story B was identical in the repeated and nonrepeated versions. In addition, Story B was semantically unrelated to Story A; the two stories did not share a story line or characters. However, similar to Story A, Story B described a message writer, an addressee, and a note. The introduction described the interaction between the two characters. This was followed by the critical phrase, which was a note that the message writer left for the addressee. This was identical to the critical phrase from the repeated version of Story A. However, the phrase was presented in a neutral context and was therefore ambiguous; it was unclear whether the note was intended to be sarcastic or sincere. This was followed by a conclusion, which was unrelated to the critical phrase and served to wrap up the story. Note that for each of the 14 experimental items, a different critical information section was used (e.g., "Thanks so much for all your advice"; "The place sure does look trendy"; "That local talent is something else"). (To see the full set of materials, go to people.hofstra.edu/Faculty/ Kristin_M_Weingartner/appendices.html.)

The dependent measure was the response to a true-false statement that followed the conclusion in Story B. The statement was consistent with a sarcastic interpretation of the critical phrase. Therefore, if readers interpret the critical phrase in Story B as sarcastic, they should respond "true"; if they interpret the critical phrase as sincere, they should respond "false."

Twenty-six filler items were also presented. Fifteen of the fillers had a phrase in quotes to make them similar to the experimental items. However, in order to minimize the number of ambiguous phrases in the experiment, we made sure that none of the phrases in quotations were ambiguous. Furthermore, because no true-false statement followed Story A in the experimental items, three of the filler passages were also not followed by a true-false statement. In addition, in contrast to the experimental items for which there was no objectively correct answer to the true-false statements, for all of the filler passages, the correct answer could be easily inferred from information provided. Finally, because we assumed that for the experimental items, the default interpretation of the ambiguous critical phrases would be a literal, or sincere, interpretation, we expected there would be more "false" than "true" responses. To offset this anticipated imbalance in responding, we constructed the filler items so that the correct answer was "true" more often than "false." Specifically, for 17 of the 23 filler items with true-false statements, the correct answer was "true."

Design. For each participant, experimental items were randomly assigned to the repeated and nonrepeated versions with two constraints: (1) Each participant read half of the experimental items in each ver- sion, and (2) each version was presented to half of the participants. Filler items were interspersed among the experimental items, and the order of presentation was the same for all of the participants.

Procedure. The participants were tested individually in sessions that lasted approximately $45 \mathrm{~min}$. They were instructed to read each story for comprehension. The items were presented on a computer monitor. The participants controlled the presentation of the text with a line advance key on a response box. Each keypress caused the current line of text to be erased and the next line to be presented. Between stories, the phrase "Press Advance For the Next Story" appeared at the center of the monitor. Importantly, this phrase appeared between Story A and Story B in all of the experimental items. That is, readers had no reason to treat Story A and Story B as part of the same experimental trial. Immediately after the last line of Story B, and after the last line of 23 of the 26 filler items, a keypress caused the current line to be erased and the string "XXX" to appear in the center of the screen. Participants did not know they were at the end of the story until this cue appeared. After $500 \mathrm{msec}$, this string was replaced by the true-false statement. Participants were instructed to respond to the statement as quickly and as accurately as possible by pressing a TRUE or FALSE key on the response box. Participants were instructed that for some of the stories there was no correct answer, and in those cases they should just provide their best guess. Following the participants' response, the true-false statement was erased from the screen. On trials in which participants took longer than 2,500 msec to respond, the phrase "PLEASE TRY TO RESPOND TO QUESTIONS MORE QUICKLY" was presented for 2,500 msec. The participants were given two breaks during the experiment. To familiarize the participants with the procedure, they were given three practice items before beginning the experiment.

\section{Results and Discussion}

We performed statistical analyses both with participants as a random effect variable $\left(t_{1}\right)$ and with items as a random effect variable $\left(t_{2}\right)$. We used an alpha level of .05 for all analyses. The results for Experiment 1 are presented in Table 1. Most importantly, the pattern of "true" responses differed significantly for the repeated and nonrepeated versions: As predicted, participants were more likely to interpret the critical phrase in Story B as sarcastic in the repeated version. They responded "true" $18.3 \%$ of the time in the repeated version versus $11.4 \%$ of the time in the nonrepeated version (the 95\% confidence interval for the $6.9 \%$ difference: 1.1 to 12.6). Another way of describing the data is that, of the "true" responses, $62 \%$ were made in the repeated version versus only $38 \%$ in the nonrepeated version. This difference was reliable by participants $\left[t_{1}(53)=2.75, S E M=.175\right]$ and by items $\left[t_{2}(13)=2.59\right.$, SEM $\left.=.72\right]$.

As was predicted, readers responded "false" for the majority of the experimental items: Averaging over the two versions, approximately $85 \%$ of the responses were "false." This indicates that the critical phrase in Story B was usually interpreted as sincere. This is not surprising, assuming that without any context to guide their interpretations, readers generally interpreted the critical phrase as sincere, at least when asked explicitly about their interpretation. Given this, evidence of a difference in the pattern of responses across the two versions is all the more impressive: Even though readers most often interpreted the critical phrase in Story B to be sincere, the information they retrieved from their memory representation of Story A significantly influenced comprehension, leading them to find the otherwise sincere-sounding critical phrase to be sarcastic, at least some of the time. 
Table 1

Experiment 1: Responses to True-False Statement

and Conclusion Line Reading Times (Story B)

\begin{tabular}{|c|c|c|c|c|}
\hline \multirow[b]{2}{*}{ Version } & \multirow{2}{*}{$\begin{array}{c}\text { "True" } \\
\text { Responses }\end{array}$} & \multicolumn{2}{|c|}{$\begin{array}{l}\text { Response Time } \\
(\mathrm{msec})\end{array}$} & \multirow{2}{*}{$\begin{array}{c}\text { Reading Time, } \\
\text { Conclusion Line (msec }\end{array}$} \\
\hline & & True & False & \\
\hline Repeated & 18.3 & 1,555 & 1,450 & 1,994 \\
\hline Nonrepeated & 11.4 & 1,390 & 1,487 & 1,800 \\
\hline Difference & $6.9^{*}$ & & & $194^{*}$ \\
\hline
\end{tabular}

True-false response times. Although the participants were somewhat slower to respond "true" in the repeated version than in the nonrepeated version, this difference was not reliable [1,555 vs. $1,390 \mathrm{msec}$, respectively, $p \mathrm{~s}>$ .15]. The time to respond "false" was similar across versions: $1,450 \mathrm{msec}$ for the repeated version and $1,487 \mathrm{msec}$ for the nonrepeated version $(p s>.2)$.

Conclusion line reading times. Although the conclusion line in Story B was identical for the two versions, reading times on this line were $194 \mathrm{msec}$ slower in the repeated version than in the nonrepeated version $(1,994$ vs. $1,800 \mathrm{msec}$, respectively). This 194-msec difference ( $95 \%$ confidence interval: 107 to 281) was reliable by subjects $\left[t_{1}(53)=4.49, S E M=43.3\right]$ and by items $\left[t_{2}(13)=3.14\right.$, $S E M=60.9]$. It is not entirely clear what this reading time difference reflects; however, a couple of possibilities exist. First, assuming that a sincere interpretation is the default, or the dominant interpretation, interpreting it as sarcastic may have demanded additional processing. For example, readers may have initially activated a sincere interpretation and then revised this on the basis of the reactivated information from Story A. Although there was no slowdown on the critical phrase itself (1,895 vs. $1,915 \mathrm{msec}$ for the repeated vs. nonrepeated versions, respectively), it is possible that any additional processing associated with activating the sarcastic, or subordinate, meaning occurred toward the end of the critical phrase and thus did not show up until the conclusion line. A second possibility is that the slowdown on the conclusion line was simply the result of the anomaly of encountering a repeated phrase in the repeated version, rather than the result of any additional processing needed to interpret the phrase in its sarcastic meaning. These possibilities are discussed further in Experiments 2 and 3 .

In summary, the results of Experiment 1 provide evidence that at least under some conditions, readers reactivate repeated information from prior, unrelated stories. After reading the critical phrase in Story B, readers retrieved this phrase, and its interpretation, from their memory representation of Story A. The reactivated information then led readers to be more likely to interpret the critical phrase in Story B sarcastically, even though it was presented in a neutral context. This occurred despite the fact that participants had no reason to believe that subsequent stories were related to each other and despite the fact that the stories within an experimental item did not share characters or a theme.

Although the results of Experiment 1 provide initial evidence of transfer effects between unrelated passages, the scope of our conclusions is limited by the methodology.
Asking participants to read a story and then answer questions about their interpretation of it is likely to involve processes that are absent from natural reading. For example, the presence of the true-false statement might have encouraged readers to reflect upon their interpretation of the critical phrase in ways they would not have, under ordinary circumstances. To remedy this, in the subsequent experiments, we ask whether transfer effects are found in conditions that more closely resemble natural reading.

\section{EXPERIMENT 2}

In Experiment 2, we used the same two versions of the passages from Experiment 1. However, instead of having participants make explicit judgments about their interpretation of the critical phrase, in the present experiment the dependent measure was reading time on a target line that was consistent with a sincere interpretation of the critical phrase in Story B. Consider the sample item in the Appendix. After the participant read the critical phrase "thanks so very much for all your advice," the sincere-consistent target line was: "Laura was relieved that Brian appreciated her advice." This line is sensible only if readers interpreted the critical phrase as sincere. Thus, if readers interpreted the phrase as sarcastic, the sincere-consistent information in the target line should be difficult to integrate, leading to a slowdown in reading.

In addition to eliminating the secondary task from Experiment 1, we revised the nonrepeated version somewhat. Because the dependent variable was reading time, we better equated the critical information sections in the two versions: In the nonrepeated version, the message writer now comments on her interaction with the addressee, but instead of having had a negative experience, as she did in the repeated version, the experience was positive. For example, in the nonrepeated version of the sample item, Valerie appreciates Eric's reminders.

In summary, if transfer effects occur across unrelated passages, even in the absence of a secondary task, readers should again interpret the critical phrase in Story B as sarcastic more often in the repeated version than in the nonrepeated version, leading to longer reading times on the sincere-consistent target line.

\section{Method}

Participants. A total of 90 undergraduates at the State University of New York at Binghamton participated as part of the requirement for an introductory psychology course. The data from 8 participants who did not follow instructions were eliminated. Therefore, our analyses were based on the data of 82 participants. 
Materials. We used the same 14 experimental items and 26 filler items from Experiment 1, with several modifications (see the Appendix). The first modification was that we eliminated the true-false statement following the critical phrase in Story B. In its place, we added a sincere-consistent target line. This line made it clear that the addressee had interpreted the critical phrase as sincere. Second, we added a posttarget line and a conclusion, to wrap up the story. And finally, to better equate the two versions, in Story A, we rewrote the critical information section in the nonrepeated version so that it was clear the message writer felt positively about the event described in the introduction.

For the filler items, we also eliminated the true-false statements. Immediately after the last line of Story B, in the experimental items, and at the end of 19 of the 26 filler items, we presented a comprehension question to ensure that the participants were reading carefully. For half of the comprehension questions, the correct answer was "yes."

Design. The design was identical to that used in Experiment 1.

Procedure. The procedure was identical to that in Experiment 1 except that the participants were not presented with a true-false statement at the end of each item. However, they were presented with a comprehension question at the end of Story B and after 19 of the 26 filler items.

\section{Results}

The mean reading times for the target and posttarget lines are presented in Table 2. We calculated these values after discarding outliers (Tukey, 1977); this eliminated $2.8 \%$ of the data. Consistent with the finding of a repetition effect in Experiment 1, reading times on the sincere-consistent target line were slower in the repeated version than in the nonrepeated version $(2,194$ vs. 2,113 msec, respectively). This $81-\mathrm{msec}$ difference (95\% confidence interval: 16.5 to 145.8 ) was reliable by participants $\left[t_{1}(81)=2.5, S E M=32.5\right]$ but not by items $\left[t_{2}(13)=1.43, S E M=51.0, p=.18\right]$. Furthermore, the slowdown in the repeated version continued onto the posttarget line. The 61-msec posttarget line difference was reliable by participants $\left[t_{1}(81)=1.99, S E M=30.5\right]$ but not by items $\left[t_{2}(13)=1.07, S E M=56.5, p=.30\right]$. Despite the target and posttarget line differences in Story B, there were no reading time differences on the critical phrase, as can be seen in Table 3. Finally, to check for order effects, we examined the correlation between effect size (reading

Table 2

Experiments 2, 3, and 4: Target Line and Posttarget Line Reading Times (in Milliseconds)

\begin{tabular}{ccc}
\hline \multicolumn{1}{c}{ Version } & Target Line & Posttarget Line \\
\hline \multicolumn{2}{c}{ Experiment 2: Sincere-Consistent Target Line } \\
Repeated & 2,194 & 1,867 \\
Nonrepeated & 2,113 & 1,806 \\
Difference & $81^{* *}$ & $61^{* *}$ \\
\multicolumn{1}{c}{ Experiment 3: Sarcasm-Consistent Target Line } \\
Repeated & 2,585 & 2,223 \\
Nonrepeated & 2,740 & 2,225 \\
Difference & $-155^{*}$ & -2 \\
\multicolumn{2}{c}{ Experiment 4: Sarcasm-Consistent Target Line } \\
Repeated & 2,356 & 1,932 \\
Nonrepeated & 2,383 & 1,925 \\
Difference & -27 & 7 \\
\hline
\end{tabular}

${ }^{*} p<.05$ (participants and items analyses). ${ }^{* *} p<.05$ (participants analysis only).
Table 3

Experiments 2, 3 and 4: Story B Critical Phrase Reading Times (in Milliseconds)

\begin{tabular}{lccc}
\hline \multicolumn{1}{c}{ Version } & Experiment 2 & Experiment 3 & Experiment 4 \\
\hline Repeated & 2,095 & 1,994 & 1,808 \\
Nonrepeated & 2,147 & 1,969 & 1,784 \\
Difference & -52 & 26 & 24 \\
\hline
\end{tabular}

Note-For all difference scores, $p>.3$ (participants and items analyses).

time on the target line for the repeated version minus reading time on the target line for the nonrepeated version) and passage number. This was close to zero $(r=-.028)$.

Consistent with the conclusions from Experiment 1, there was a repetition effect in the present experiment. The initial presentation of the critical phrase in Story A in a sarcastic context led readers to be more likely to interpret the critical phrase in Story B as sarcastic in the repeated version. This occurred even though the phrase appeared in a neutral context in Story B and even though the two stories were unrelated. Furthermore, in contrast to Experiment 1, readers were not asked explicitly about their interpretation of the critical phrase.

Although the results of both Experiments 1 and 2 provide evidence of a repetition effect across unrelated stories, the results of Experiment 2 leave a number of questions unanswered. First, the difference between versions was not significant in the items analysis. Second, in Experiment 1 , reading times on a neutral postcritical message line in Story B (the conclusion line) were slower in the repeated version than in the nonrepeated version, due, presumably, to some type of processing spillover from the critical phrase. Given this, it is possible that the slowdown we found on the target line in the repeated version of the present experiment was due at least in part to this type of spillover effect. To provide a stronger test of the influence of repeated information in unrelated stories, in Experiment 3 we used a sarcasm-consistent target line instead of a sincere-consistent target line.

\section{EXPERIMENT 3}

The materials in Experiment 3 were identical to those in Experiment 2 except that the target line was consistent with a sarcastic interpretation of the critical message. Consider the sample item in the Appendix. In Story B, after the critical phrase "And thanks so very much for all your advice," the target line was: "He was irritated that she always gave him advice." If comprehension of the critical phrase in Story B is influenced by its prior presentation, then, as in Experiments 1 and 2, readers should be more likely to interpret it as sarcastic in the repeated version than in the nonrepeated version. However, in contrast with Experiment 2, reading times should now be faster, rather than slower, on the target line in the repeated version. This design eliminates the concern that there might be a slowdown associated with encountering a repeated phrase. Any slowdown on the target line in the repeated version would work against the predicted effect. 


\section{Method}

Participants. A total of 33 undergraduates at the State University of New York at Binghamton participated as part of the requirement for an introductory psychology course. The data from 3 participants who did not follow instructions were eliminated. Therefore, our analyses were based on the data from 30 participants.

Materials. We used the same 14 experimental and 26 filler items used in Experiment 2, with the following modifications: We replaced the sincere-consistent target line in Story B with a sarcasmconsistent target line. This line made it clear that the addressee had interpreted the message as sarcastic. In addition, we rewrote the posttarget and conclusion lines so that they followed smoothly.

Design and Procedure. The design and procedure were identical to those used in Experiment 2.

\section{Results}

The mean reading times for the target and posttarget lines are presented in Table 2. We calculated these values after discarding outliers (Tukey, 1977); this eliminated $1.9 \%$ of the data. Consistent with our conclusions in Experiments 1 and 2, reading times on the sarcasmconsistent target line were faster in the repeated version than in the nonrepeated version $(2,585$ vs. $2,740 \mathrm{msec}$, respectively). This 155 -msec difference ( $95 \%$ confidence interval: 5.4 to 304.5$)$ was significant by participants $\left[t_{1}(29)=2.1, S E M=73.1\right]$ and by items $\left[t_{2}(13)=2.5\right.$, $S E M=67.1]$. There was no reading time difference on the posttarget line $(p \mathrm{~s}>.8)$. As in Experiment 2, there were no order effects, with the correlation between effect size and passage number being close to zero $(r=-.06)$, and no reading time differences on the critical phrase, as can be seen in Table 3 .

In addition to providing converging evidence for a repetition effect across unrelated stories, the results of this experiment rule out a number of concerns raised in Experiment 2. First, the effect is reliable by items. Second, any slowdown caused by the novelty of encountering a repeated phrase in Story B, or by the additional processing needed to interpret the critical phrase in its sarcastic, subordinate meaning, should have led to slower, rather than faster, reading times on the target line in the repeated version. Given this, the finding that reading times were faster in the repeated version than in the nonrepeated version, in combination with the results of Experiments 1 and 2, provides convincing evidence that the memory representation of the critical phrase was reactivated when readers processed Story B.

\section{EXPERIMENT 4}

The goal of Experiment 4 was to examine an alternative explanation for our findings: Although the first three experiments make it clear that in the repeated version, readers' interpretation of the critical phrase in Story B was influenced by the sarcastic critical phrase in Story A, it is unclear whether readers reactivated from memory the meaning of the critical phrase from Story A or whether they simply reactivated an awareness that phrases can be interpreted sarcastically. That is, in the first three experiments, there were actually two ways in which the repeated version differed from the nonrepeated version: (1) In the repeated version, a phrase was repeated from Story A to Story B, and (2) in the repeated version, there was a sarcastic phrase in Story A. Although we have suggested that it was the overlap in the linguistic content of the phrases that was essential, in the present experiments we ask whether simply having sarcasm present in Story A might have led to a sarcastic interpretation of the critical phrase in the repeated version of Story B.

To examine this alternative explanation, we used the same materials that were used in Experiment 3, except that we paired Story A from one passage with Story B from a different passage. For example, Story A from Passage 1 was paired with Story B from Passage 8 . Consider the sample passage. Although there is a sarcastic phrase in the repeated version of Story A ("Thanks so very much for all your advice."), it does not match the critical phrase in Story B ("Mind helping you move furniture? Why would I possibly mind?"). If readers are more likely to interpret the critical phrase in Story B as sarcastic simply because sarcasm is present in Story A, then reading times on the sarcasm-consistent target line should be faster in the repeated version than in the nonrepeated version, replicating the results of Experiment 3. In contrast, if the repetition effects found in the first three experiments were due to readers' reactivating the meaning of the critical phrase from Story A, there should be no difference between versions, because neither version contains a repeated phrase in Story B.

\section{Method}

Participants. A total of 38 undergraduates at the State University of New York at Binghamton participated as part of the requirement for an introductory psychology course. The data from 3 participants who did not follow instructions were eliminated. Therefore, our analyses were based on data from 35 participants.

Materials. We used the same 14 experimental and 26 filler passages from Experiment 3 in this experiment. The only modification was in the order of presentation. For example, Story A from Passage 1 in Experiment 3 was followed by Story B from Passage 8, and so on. Given this, participants saw the identical set of passages as in Experiment 3, but phrases were not repeated within a passage pair.

Design and Procedure. The design and procedure were identical to those used in Experiments 2 and 3.

\section{Results}

The mean reading times for the target and posttarget lines are presented in Table 2. We calculated these values after discarding outliers (Tukey, 1977); this eliminated $2.7 \%$ of the data. In contrast with the reliable 155-msec difference found in Experiment 3, reading times on the sarcasmconsistent target line did not differ in the repeated and nonrepeated versions: 2,356 versus $2,383 \mathrm{msec}$, respectively [95\% confidence interval: -147.9 to $93.6, t_{1}(34)=$ $.46, S E M=59.4$ and $\left.t_{2}(13)=1.4, S E M=62.6\right]$. There were also no reading time differences on the posttarget line $(p s>.6)$ or on the critical phrase (see Table 3).

The results of this experiment demonstrate that simply having sarcastic information present in Story A is not sufficient to lead readers to a sarcastic interpretation of the critical phrase in Story B. A sarcastic interpretation of the 
critical phrase in Story B occurs only when the identical phrase is used sarcastically in Story A. Thus, we can conclude that in Experiments 1, 2, and 3, when readers processed the critical phrase in Story B, they reactivated the meaning of the critical phrase in Story A, and not simply the fact that sarcastic information was present.

\section{GENERAL DISCUSSION}

Despite the extensive evidence demonstrating that rereading benefits are limited to conditions in which the context remains consistent across the two processing episodes (e.g., Levy et al., 1995), in the present set of experiments, we asked whether text repetition effects are ever found across thematically and semantically unrelated narratives. Given what we know about memory in general, and assuming that text inputs act like any other input to memory, we hypothesized that there should be at least some conditions in which concepts from one narrative serve to reactivate concepts from another narrative.

In the present set of experiments, we attempted to select a set of conditions in which we were most likely to observe a rereading benefit across unrelated narratives. This was done in an attempt to establish some boundary conditions. Most significantly, we created materials in which a whole phrase was repeated across passages rather than only a single word. In three experiments, using both an offline measure (Experiment 1) and an online measure (Experiments 2 and 3), we found evidence of rereading benefits for the repeated phrases even though the participants were focused on comprehending the stories rather than on the individual words or phrases, and even though they had no reason to treat the stories as related. Furthermore, Experiment 4 ruled out the possibility that it was simply an awareness of sarcasm that was driving the effect. Taking all this together, we can conclude that when readers processed the critical phrase in the repeated version of Story B, it led to the reactivation from memory of the critical phrase from Story A. Furthermore, because the critical phrase was initially comprehended as sarcastic, the sarcastic interpretation was retrieved from memory as well. Not surprisingly, readers' memory representation contained more than the surface features - that is, the actual words of the critical message - and also included their understanding of that phrase.

In what way might the reactivation of the critical phrase from Story A have influenced comprehension of the critical phrase in Story B? Most simply, the reactivation of the sarcastic meaning of the phrase during the reading of the phrase in Story B increased the probability that readers would interpret the phrase as sarcastic, despite its presentation in a neutral context and despite the dominance of the literal, sincere interpretation. However, another possibility exists, at least for Experiment 3: The retrieval of the critical phrase from memory may not have led readers to initially interpret the critical phrase in Story B as sarcastic, but it may have facilitated a reinterpretation on the target line. That is, in both the repeated and nonrepeated versions, the sarcasm-consistent target line may have initially been perceived as anomalous. However, in the repeated version, readers may have used the reactivated sarcastic interpretation to reinterpret the critical phrase and to repair the anomaly, leading to faster reading times than in the nonrepeated version. In other words, it is possible that the retrieval of the phrase from memory did not influence comprehension initially but played a role only after readers processed the anomalous target line.

Despite this possibility, the argument that the repeated phrase has its effect only during target line reading cannot be made for Experiment 2. Because the target line in Experiment 2 was consistent with a sincere interpretation of the critical phrase, if readers did not make use of the reactivated sarcastic interpretation of the phrase during the reading of the critical phrase itself, there would have been no reason for them to make use of it during target line reading. Given this, the most parsimonious explanation for the full set of findings is that readers reactivated the critical phrase from memory while they were processing the critical phrase in Story B, and the reactivated memory traces immediately influenced comprehension. Importantly, regardless of when the reactivated information had its influence, we can confidently conclude that readers reactivated the critical phrase and its interpretation from memory during rereading, despite the fact that the stories were thematically and semantically unrelated.

What text factors might have contributed to our findings? That is, why did we find rereading effects with unrelated passages when prior studies have not? First, and probably most importantly, in contrast with previous rereading studies, we examined the processing of repeated phrases rather than the processing of repeated words. We assume that repeated phrases are more effective retrieval cues than repeated words, in part because repeated phrases provide more overlap across the two processing episodes. In addition, the effectiveness of a memory retrieval cue should depend in part on the amount of attention readers pay to that cue. We assume that phrases receive more attention than single words. Most simply, a phrase is a larger input to memory than a single word, consisting of at least one, and sometimes several, propositions.

An additional change from previous rereading studies is that the repeated text was ambiguous. It is possible that the ambiguity of the phrases also led to an increase in the amount of attention that they received. This seems particularly plausible given the subordinate, sarcastic interpretation of the critical phrase in Story A; readers may have settled on this interpretation in the repeated version only after the literal meaning had been rejected. Consistent with this idea, there is some evidence that figurative meanings are accessed more slowly than their literal counterparts (e.g., Gibbs, 1990; Onishi \& Murphy, 1993).

Note that Carr and Brown (1990) and Carlson et al. (1991) also emphasized the role of attention in predicting when repetition effects should be found. According to the level-of-focal-attention hypothesis (Carr \& Brown, 1990), when readers attend to processing individual lexical items, either because of the nature of the experimental instructions or because of the nature of the task itself (e.g., speeded oral reading), there will be a large contribution from abstract, or context-independent, processing. 
In these cases, the repetition of a word in a mismatching context during test may be sufficient to produce a repetition effect.

However, in contrast to tasks that emphasize word-level processing, in our experiments readers were focused on text-level comprehension, and thus, given the level-offocal-attention hypothesis, readers would be expected to form representations that were context dependent, or episodic. But perhaps the nature of our manipulation led readers to pay particular attention to the individual words making up the critical phrases: The phrases were in quotes, they conveyed a figurative, sarcastic meaning, and so on. As Carr and Brown (1990) argued, "one must take seriously the possibility that processing will exhibit both abstraction and episodic sensitivity" and that this "may change with stimulus properties" (p. 732). Along with this, perhaps readers sometimes focus their attention on the word level, even within a comprehension task, if they encounter lexical items that are salient-a lowfrequency word, a figurative expression such as an idiom or a sarcastic phrase, or perhaps even dialogue with strong emotional valence. With this additional assumption, our claims can be framed within the level-of-focal-attention hypothesis: Because of the salience of the critical phrases, when readers processed them, they attended not only to the ongoing comprehension of the passage but also to the particular lexical items making up the phrases. Thus, the phrases were able to be retrieved from memory even when the retrieval cue (i.e., the critical phrase in Story B) was presented in a mismatching context.

Whether, in fact, the salience of the critical phrases led readers to represent them in a way that was qualitatively different - on a word level rather than on a text level (Carr \& Brown, 1990), or on a surface level rather than a situationmodel level (Raney, 2003) - is difficult to determine. It might instead be argued that the salience of the critical phrases led to a quantitative difference in their representation: Because the critical phrases were salient, they were more strongly attended to, and thus, they were more likely to be encoded into memory, and likely to be more richly encoded into memory, making them easier to retrieve. Differentiating between these two explanations is not easy. For one thing, it might be argued that one way to richly encode information is to include lexical, or surface-level, information in the representation. However, regardless of the particular consequences of the increased attention to the critical phrases, attention is an important factor in explaining our results.

Although Raney's (2003) context-dependent representation model can account for the effects of attention, our results are inconsistent with a central prediction of the model: "When situation models are well developed but there is no semantic overlap between them . . little or no repetition benefit is expected" (p. 22). Even though readers in our experiments were focused on the comprehension of the passages, or on building a situation model, there was strong evidence of rereading effects across different contexts. Not only wasn't the context consistent across the two processing episodes, it changed fairly dramatically: Story A and Story B were thematically distinct - they did not share a story line, nor did they have any story characters in common. Thus, although there is evidence demonstrating that repetition effects are sometimes limited to conditions in which the context remains the same across presentations (e.g., Levy et al., 1995), our results demonstrate that rereading effects can be found across unrelated episodes, at least under some conditions. Minimally, these results call for some modifications to the context-dependent representation model.

Perhaps the best way to explain our findings, as well as the results from all of the rereading studies, is to appeal to basic memory principles. Given this approach, we would argue that the extent to which a word or phrase reactivates concepts from a same versus different episode should be dependent on the same complex set of factors that influences memory retrieval more generally: attention, the strength of the overlap between the two processing episodes, the amount and type of intervening material, and so on. More specifically, consider Myers and O'Brien's (1998) resonance model of text processing: Text inputs act like any other input to memory, automatically activating related information in memory. All related concepts in memory resonate in parallel (cf. Gillund \& Shiffrin, 1984; Hintzman, 1986; Ratcliff, 1978), those from episodic memory as well as those from semantic memory. The degree to which concepts resonate depends on their level of activation as well as the number of semantic and conceptual features that overlap with the current text input. Given this, concepts from semantic memory, in this case the previously read passage, should become reactivated if the conditions are optimal. Thus, if a text input overlaps with concepts from a previous narrative, the probability that those concepts will be retrieved should increase if they have been elaborated extensively, if they are particularly salient and memorable, if the featural overlap with the current input is sufficiently strong, if the current text input receives a significant amount of attention, and so on.

How might this framework account for the evidence demonstrating the reduction of repetition benefits when the context is changed between the two presentations (e.g., Levy et al., 1995)? Consider Myers and O’Brien's (1998) argument that in most conditions, "resonating concepts will be dominated by those in the episodic memory trace" simply because there are more related concepts from within a narrative than from anywhere else in memory. That is, given that narratives are organized around a set of characters, locations, and so on, there should be extensive overlap between different segments of a narrative, leading to a representation that is highly interconnected.

In addition to the conceptual and semantic overlap's being more extensive within passages than between them, in some cases each passage input may be connected in memory to some sort of a passage, or context marker, such as a title, further increasing the interconnection of passage inputs as well as the probability that a text element will lead to the reactivation of other inputs from the same, rather than a different, passage. Given this, we can expect that repetition effects will often be limited or nonexistent 
between unrelated passages, as has been found in the past, and as is predicted by Raney's (2003) context-dependent representation model. However, within a memory-based framework, this can be explained as being due to the lack of overlap between passages rather than to the memory representation of an individual passage being somehow informationally encapsulated or episodically impenetrable.

Although the memory-based framework and Raney's (2003) context-dependent representation model make some overlapping predictions, we believe that there are a number of advantages to framing the rereading findings within the resonance model. First, and most simply, it provides an explicit process model. Second, it provides an excellent account of our findings: Whereas a central prediction of the context-dependent representation model is that little or no rereading benefit should be found between unrelated passages when a situation model is developed, according to a memory-based framework, we should expect retrieval of information from outside the episodic representation in a number of conditions, such as when readers pay particular attention to an input. More generally, a fundamental characteristic of a memory-based framework such as Myers and O’Brien's (1998) resonance model is that memory retrieval is influenced by a complex interaction of a large set of factors, making our results unsurprising. That is, although repetition effects may be less probable across unrelated contexts than across related contexts, the influence of context should interact with factors such as the salience of the repeated information, as we have shown. A third advantage of a memory-based approach is that it eliminates the need for discourse-specific constructs such as situation models, which are often ill defined. Instead, this framework appeals primarily to basic constructs in memory and attention, providing an elegant bridge between research areas.

Finally, although we developed one set of conditions in which a rereading effect was found across unrelated passages, clearly there are other conditions in which this would not occur. The question is, what factors were necessary for our findings? For example, if the critical repeated phrases were unambiguous, would we have found repetition effects? What if the critical phrases were biased toward their dominant, nonsarcastic meaning in Story A, instead of their subordinate, sarcastic meaning? What if Story A had been an expository text and Story B had been a narrative, making the context change even greater? What if an unrelated passage intervened between Story A and Story B; would repetition effects still be found? Clearly, to develop a complete theory of rereading, and more generally to develop an understanding of the nature of a reader's memory representation, an understanding is needed of the influence of a whole range of stimulus and reader characteristics on the nature of the text representation.

\section{AUTHOR NOTE}

Portions of this research were presented at the 16th Annual Meeting of the Society for Text and Discourse (2006, Minneapolis, MN). We thank Debbie Berk, Gina Choi, Meredith Jones, Ryan Kinney, Natalie Lusher, Robyn Mierzejewski, and Anna Taran for their assistance in data collection. Correspondence concerning this article should be addressed to C. M.
Klin, Department of Psychology, State University of New York at Binghamton, Binghamton, NY 13902-6000 (e-mail: cklin@binghamton.edu).

\section{REFERENCES}

Besson, M., \& Kutas, M. (1993). The many facets of repetition: A cued-recall and event related potential analysis of repeating words in same versus different sentence contexts. Journal of Experimental Psychology: Learning, Memory, \& Cognition, 19, 1115-1133.

Carlson, L., Alenjano, A., \& Carr, T. H. (1991). The level of focal attention hypothesis in oral reading: Influences of strategies on the context specificity of lexical repetition effects. Journal of Experimental Psychology: Learning, Memory, \& Cognition, 17, 924-931.

CARr, T. H., \& Brown, J. S. (1990). Perceptual abstraction and interactivity in repeated oral reading: Where do things stand? Journal of Experimental Psychology: Learning, Memory, \& Cognition, 16, 731-738.

Carr, T. H., Brown, J. S., \& Charalambous, A. (1989). Repetition and reading: Perceptual encoding mechanisms are very abstract but not very interactive. Journal of Experimental Psychology: Learning, Memory, \& Cognition, 15, 763-778.

Gerrig, R. J., \& McKoon, G. (1998). The readiness is all: The functionality of memory-based text processing. Discourse Processes, 26, 67-86.

GiBBs, R. W. (1990). Comprehending figurative referential descriptions. Journal of Experimental Psychology: Learning, Memory, \& Cognition, 16, 56-66.

Gillund, G., \& Shiffrin, R. M. (1984). A retrieval model for both recognition and recall. Psychological Review, 91, 1-67.

HaYMan, C. A. G., \& JACOBY, L. L. (1989). Specific word transfer as a measure of processing in the word-superiority paradigm. Memory \& Cognition, 17, 125-133.

Hintzman, D. L. (1986). "Schema abstraction" in a multiple-trace memory model. Psychological Review, 93, 411-428.

JACOBY, L. L. (1983). Remembering the data: Analyzing interactive processes in reading. Journal of Verbal Learning \& Verbal Behavior, 22, 485-508.

JACOBY, L. L., BAKeR, J. G., \& BrooKs, L. (1989). Episodic effects on picture identification: Implications for theories of concept learning and theories of memory. Journal of Experimental Psychology: Learning, Memory, \& Cognition, 15, 275-281.

LeVy, B. A., \& Burns, K. I. (1990). Reprocessing text: Contributions from conceptually driven processes. Canadian Journal of Psychology, 44, 465-482.

Levy, B. A., Campsall, J., Browne, J., Cooper, D., Waterhouse, C., \& Wilson, C. (1995). Reading fluency: Episodic integration across texts. Journal of Experimental Psychology: Learning, Memory, \& Cognition, 21, 1169-1185.

LEVy, B. A., \& KirSner, K. (1989). Reprocessing text: Indirect measures of word and message level processes. Journal of Experimental Psychology: Learning, Memory, \& Cognition, 15, 407-417.

MacLeod, C. M. (1989). Word context during initial exposure influences degree of priming in word fragment completion. Journal of Experimental Psychology: Learning, Memory, \& Cognition, 15, 398-406.

Masson, M. E. J., \& Freedman, L. (1990). Fluent identification of repeated words. Journal of Experimental Psychology: Learning, Memory, \& Cognition, 16, 355-373.

McKoon, G., \& Ratcliff, R. (1995). The minimalist hypothesis: Directions for research. In C. A. Weaver, S. Mannes, \& C. R. Fletcher (Eds.), Discourse comprehension: Essays in honor of Walter Kintsch (pp. 97-116). Hillsdale, NJ: Erlbaum.

Morton, J. (1969). Interaction of information in word recognition. Psy chological Review, 76, 165-178.

Myers, J. L., \& O'Brien, E. J. (1998). Accessing the discourse representation during reading. Discourse Processes, 26, 131-157.

O'Brien, E. J., \& Albrecht, J. E. (1992). Comprehension strategies in the development of a mental model. Journal of Experimental Psychology: Learning, Memory, \& Cognition, 18, 777-784.

OLIPHANT, G. W. (1983). Repetition and recency effects in word recognition. Australian Journal of Psychology, 35, 393-403.

Onishi, K. H., \& Murphy, G. L. (1993). When metaphors are not understood as easily as literal expressions. Memory \& Cognition, 21, 763-772. 
RANEy, G. E. (2003). A context-dependent representation model for explaining text repetition effects. Psychonomic Bulletin \& Review, 10, 15-28.

RANEY, G. E., \& RAYNeR, K. (1995). Word frequency effects and eye movements during two readings of a text. Canadian Journal of Experimental Psychology, 49, 151-172.

Raney, G. E., Therriault, D., \& Minkoff, S. (2000). Repetition effects from paraphrased text: Evidence for an integrated representation model of text representation. Discourse Processes, 29, 61-81.

RAtCliff, R. (1978). A theory of memory retrieval. Psychological Review, 85, 59-108.
Rizzella, M. L., \& O'Brien, E. J. (2002). Retrieval of concepts in script-based texts and narratives: The influence of general world knowledge. Journal of Experimental Psychology: Learning, Memory, \& Cognition, 28, 780-790.

Tenpenny, P. L. (1995). Abstractionist versus episodic theories of repetition priming and word identification. Psychonomic Bulletin \& Review, 2, 339-363.

TukEY, J. W. (1977). Exploratory data analysis. Reading, MA: AddisonWesley.

VAN DIJK, T. A., \& KINTSCH, W. (1983). Strategies of discourse comprehension. New York: Academic Press.

APPENDIX

Sample Items From Experiments 1, 2, 3, and 4

\section{Story A} Sample Item From Experiment 1

Introduction: Shortly after Valerie graduated from college, she moved into an apartment with her friend Eric. Yesterday Eric, worrying that Valerie would forget that it was trash day, left her a note advising her to take out the garbage before she went out.

\section{Critical Information:}

Repeated Version (Critical Phrase):

Valerie was really irritated that Eric felt the need to leave constant reminders. She really wanted to tell him off. So after she ate breakfast, she wrote an angry note to Eric telling him she always remembered to take out the garbage, and added:

"Thanks so very much for all your advice. Sure is nice to be treated like a 10 year old."

Nonrepeated Version:

As Valerie took out the garbage she thought about how much she liked this neighborhood. It was within walking distance of the mall where she frequently shopped. Valerie went back inside and made a pot of homemade chicken soup. She left a note for Eric:

"I've left a pot of soup cooking on the stove on low heat. Can you turn it off at 10:00?"

Conclusion: Then Valerie went for a jog around her neighborhood. She was running in a big marathon next month and knew that she would need to train every day if she had any chance of making it to the finish line.

\section{Story B}

Introduction: Brian was invited to his friend Laura's birthday party. When Brian arrived, he saw Laura right away and starting chatting with her. He told her that he had a crush on one of his coworkers. He wasn't sure if the attraction was mutual. Laura told Brian that he should take a chance and ask her out. The next day Brian wrote an e-mail to Laura to let her know that she left her jacket at his house and added:

Critical Phrase: "And thanks so very much for all your advice."

Conclusion: Brian hoped Laura would get the e-mail soon.

True-False Statement: Brian did not appreciate Laura's advice.

\section{Sample Item From Experiments 2, 3, and 4}

\section{Story A: Experiments 2, 3, and 4}

Introduction: Shortly after Valerie graduated from college, she moved into an apartment with her friend Eric. Yesterday Eric, worrying that Valerie would forget about the phone bill, left her a note advising her to pay the bills as soon as they came.

\section{Critical Information:}

Repeated Version (Critical Phrase):

Valerie always paid the bills on time, and was really irritated that Eric felt the need to leave constant reminders. She really wanted to tell him off. So after she ate breakfast, she wrote an angry note to Eric about his nagging and added:

"Thanks so very much for all your advice. Sure is nice to be treated like a 10 year old."

Nonrepeated Version:

Valerie had almost forgotten that the bill was due today and was really glad that Eric had left her a reminder. She really wanted to thank him. So after she ate breakfast, she wrote a thank you note to Eric for the reminder and added: "I really do appreciate your reminders. It makes it easy to pay the bills on time."

Conclusion: Then Valerie went for a jog around her neighborhood. She was running in a big marathon next month and knew that she would need to train every day if she had any chance of making it to the finish line. 


\section{Story B: Experiment 2}

Introduction: Brian was invited to his friend Laura's birthday party. When Brian arrived, he saw Laura right away and starting chatting with her. He told her that he had a crush on one of his coworkers. He wasn't sure if the attraction was mutual. Laura told Brian that he should take a chance and ask her out. The next day Brian wrote an e-mail to Laura to let her know that he left his jacket at her house and added:

Critical Phrase: "And thanks so very much for all your advice."

Sincere-Consistent Target Line: Laura was relieved that Brian appreciated her advice.

Posttarget Line: She had only known Brian for a couple of months

Conclusion: and thought that giving him relationship advice might have been a bad idea.

\section{Story B: Experiment 3}

Introduction: Brian was invited to his friend Laura's birthday party. When Brian arrived, he saw Laura right away and starting chatting with her. He told her that he had a crush on one of his coworkers. He wasn't sure if the attraction was mutual. Laura told Brian that he should take a chance and ask her out. The next day Brian wrote an e-mail to Laura to let her know that he left his jacket at her house and added:

Critical Phrase: "And thanks so very much for all your advice."

Sarcasm-Consistent Target Line: He was irritated that she always gave him advice.

Posttarget Line: He could never tell her about anything

Conclusion: without her trying to tell him what to do.

\section{Story B: Experiment 4}

Introduction: Chris was having lunch with one of his coworkers.

They were talking about who they thought would get promoted to vice president of the company. The buzz around the office was that Grace, the president's daughter, was going to get the job. As they were reading their menus, Chris noticed that his neighbor Angela had instant messaged him. She asked if he would mind helping her move some furniture that evening. Chris wrote back:

Critical Phrase: "Mind helping you move furniture? Why would I possibly mind?"

Sarcasm-Consistent Target Line: Chris was miffed that Angela had asked for help.

Posttarget Line: Every time he needed a favor from her,

Conclusion: she claimed to be too busy.

(Manuscript received August 28, 2006;

revision accepted for publication January 17, 2007.) 